\title{
Efficient Channel Borrowing Strategy for Real-Time Services in Multimedia Wireless Networks
}

\author{
Xiaowen Wu, Member, IEEE, Kwan L. Yeung, Senior Member, IEEE, and Jianhao Hu
}

\begin{abstract}
An efficient resource sharing strategy is proposed for multimedia wireless networks. Assume the channel resource in a wireless system is partitioned into two sets: one for voice calls and one for video calls. In the proposed channel borrowing strategy, voice calls can borrow channels from those pre-allocated to video calls temporarily when all voice channels are busy. A threshold type decision policy is designed such that the channel borrowing request will be granted only if the quality of service $(\mathrm{QoS})$ requirement on video call blocking will not be violated during the duration of channel lending. An analytical model is constructed for evaluating the performance of the channel borrowing strategy in a simplified wireless system and is verified by computer simulations. We found that the proposed channel borrowing scheme can significantly reduce the voice call blocking probability while the increase in video call blocking probability is insignificant.
\end{abstract}

Index Terms-Channel borrowing strategy, performance analysis, QoS guarantee, resource sharing.

\section{INTRODUCTION}

$\mathbf{T}$ HE next generation wireless networks need to support multimedia traffic. Different types of traffic have different traffic characteristics. To effectively utilize the bandwidth resources, several bandwidth allocation schemes have been proposed for transmission multimedia traffic in wireless networks [2]-[5]. For broadband ISDN networks, techniques such as complete partitioning, mutually restricted access [9], [12]-[14] have been widely studied.

Generally speaking, a real-time video call requires a relatively large amount of bandwidth than a voice call and data traffic does not have real-time requirement but is usually bursty. To guarantee the quality of service (QoS) requirements for each type of traffic in a wireless network, bandwidth resources are usually pre-allocated to voice, video and data. For real-time voice and video traffic, the call blocking probability is commonly used as their performance measures. For data traffic, the system throughput and packet delay performances are used. To encourage resource sharing, the idle capacity allocated to video and/or voice traffic can be temporarily used for transmitting packet switched data without degrading the video/voice call blocking performance. This is known as the movable boundary scheme [8]-[10] and an example of integrating data and voice calls on an $N$-slot TDM frame is shown in Fig. 1. Each frame is divided into voice compartment and data compartment. The idle slots in voice compartment can be lent to carry data traffic.

Manuscript received August 24, 1998; revised December 6, 1999. This work is supported by CityU Direct Allocation Grant 7100082 , City University of Hong Kong.

The authors are with the Department of Electrical and Electronic Engineering, The University of Hong Kong, Hong Kong (e-mail: kyeung @eee.hku.hk).

Publisher Item Identifier S 0018-9545(00)04840-4.
When a voice call arrives, it has preemptive priority over data and thus its performance will not be affected by lending slots to data. Recently, a double movable boundary scheme is proposed [11] for satellite networks. It divides a TDM frame into three compartments instead of two, where the extra compartment, called common resource pool (CRP), is located between the voice and the data compartments. The CRP compartment has two movable boundaries, one interfaced with voice and one interfaced with data. Under certain pre-defined resource sharing rules, voice and/or data is allowed to use the channels in the CRP compartment.

Existing approaches to bandwidth sharing in wireless networks usually only consider the resource sharing between data and one type of real-time traffic, either voice or video. Since real-time traffic has preemptive priority over data, the performance of real-time traffic will not be degraded by lending some resources to data. In this paper, we consider a system that supports all three types of traffic, data, voice and video. Unlike existing approaches, we focus on resource sharing between real-time voice and video traffic. When sharing of bandwidth between voice and video calls is allowed (i.e., a voice call can borrow the pre-allocated bandwidth from video or vice versa), we must guarantee that during the bandwidth borrowing period, the call blocking performance of both types of traffic will not violate their pre-defined QoS requirements. Otherwise, the bandwidth borrowing request should be rejected.

In this paper, we propose an efficient bandwidth sharing scheme for voice and video calls. Since data traffic in general has lower priority than the voice and video calls, they can be transmitted using the idle voice and video bandwidth, e.g., adopting the movable boundary scheme [8]-[10]. In order to focus on the bandwidth sharing between voice and video calls, we assume the system under consideration only supports voice and video traffic. For the sake of presentation, we assume the system uses TDMA and the basic bandwidth unit for resource sharing is a time slot.

The organization of this paper is as follows. Section II introduces the channel borrowing strategy for voice and video calls. An analytical model is constructed in Section III for a simplified system where each borrowed video channel can carry only a single voice call. Section IV relaxes this constraint. A general channel borrowing policy for borrowing one video channel carrying multiple voice calls is designed. Then in Section V, some practical implementation issues of the proposed channel borrowing strategy is considered. Section VI summarizes the numerical and simulation results. We found that the proposed channel borrowing strategy can significantly reduce the voice call blocking probability while only insignificant increase in 


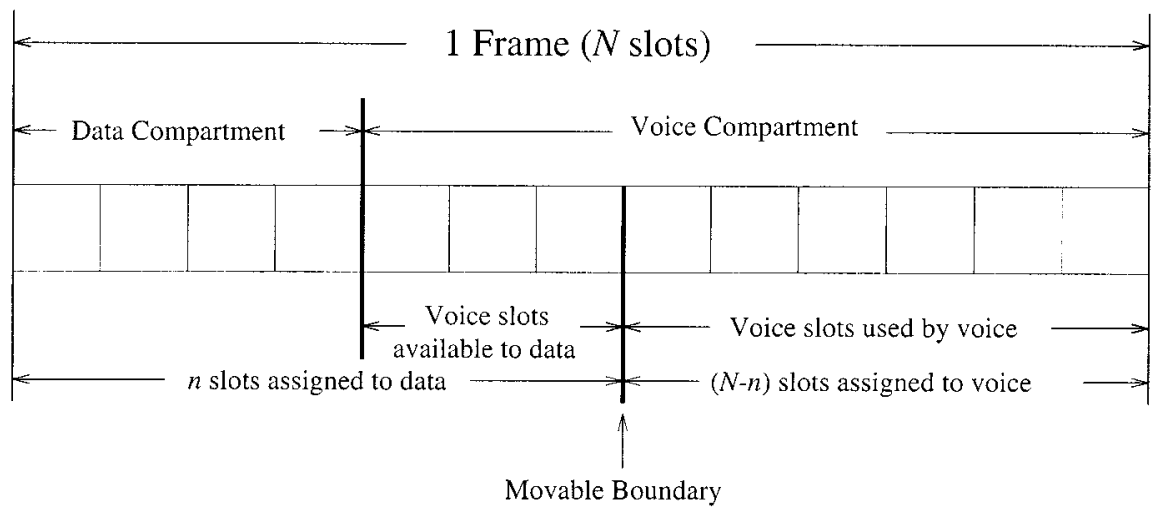

Fig. 1. Movable boundary multiplexing scheme for voice and data traffic on an $N$-slot TDM frame.

TABLE I

A SUMMARY OF MAJOR NOTATIONS USED

\begin{tabular}{c|l}
\hline$N$ & The number of pre-allocated voice channels, where a voice channel occupies 1 time slot. \\
\hline$M$ & The number of pre-allocated video channels, where a video channel occupies $n$ time slots. \\
\hline$m$ & $\begin{array}{l}\text { The number of active video calls in the system at the time of a new video channel borrowing } \\
\text { request arrives. }\end{array}$ \\
\hline$P_{B 1}$ & The voice call blocking probability with channel borrowing strategy. \\
\hline$P_{B 2}$ & The video call blocking probability with channel borrowing strategy. \\
\hline$P_{N}$ & The voice call blocking probability without channel borrowing strategy. \\
\hline$P_{M}$ & The video call blocking probability without channel borrowing strategy. \\
\hline$\lambda_{2}, \mu_{1,} \rho_{1}, \rho_{2}$ & The voice call arrival rate, voice call service rate, and voice traffic intensity. \\
\hline$P_{i}(t, m, M)$ & $\begin{array}{l}\text { The video call arrival rate, video call service rate, and video traffic intensity. } \\
\text { consideration there are m active video calls, } P_{i}(t, m, M) \text { is the probability that there are } i \\
\text { active video calls after time } t .\end{array}$ \\
\hline$P_{r}$ & The QoS requirement on video call blocking probability. (e.g. $\left.P_{r}=0.01\right)$ \\
\hline$X_{k}$ & $\begin{array}{l}\text { The maximum time that } k \text { video channels can be simultaneously borrowed without violating } \\
P_{r} .\end{array}$ \\
\hline$P_{s}$ & $\begin{array}{l}\text { The probability that the borrowed video channel can not be returned within the maximum } \\
\text { borrowing time } X_{1} .\end{array}$ \\
\hline$P_{s}{ }^{*}$ & The system requirement on $P_{s}$, i.e. $P_{s} \leq P_{s}{ }^{*}$. \\
\hline$\theta$ & The minimum channel borrowing time without violating $P_{s} \leq P_{s}^{*}$ \\
\hline have & $\begin{array}{l}\text { The probability of a successful video channel borrowing given that }(g-1) \text { video channels } \\
\text { have already been borrowed by voice calls. }\end{array}$ \\
\hline
\end{tabular}

video call blocking probability is observed. We also found that the analytical results match the simulation results very well. Finally, conclusions are presented in Section VII.

\section{Channel Borrowing StRATEgy}

Let a system be engineered such that it can simultaneously carry $N$ voice calls and $M$ video calls. A voice call occupies one time slot in a TDM frame and a video call occupies $n$ time slots. In general, video traffic is expected to have a lower call arrival rate than that of voice. Since video call requires a higher bandwidth than a voice call, borrowing bandwidth for carrying video calls is more difficult and should not be encouraged. Therefore, we assume that only voice traffic can borrow bandwidth from that pre-allocated to video. That is when a voice call arrives and found all $N$ voice channels are busy, a video channel can be borrowed to carry the voice call if the video call blocking probability requirement, denoted by $P_{r}$, will not be violated. The crucial point here is to determine if sufficient number of idle video channels are available (after lending) for preventing the video call blocking probability from exceeding its QoS during the channel lending time. This depends on the channel occupancy of the video calls at the time of lending, as well as the duration of channel lending. For convenience, Table I summarizes some main notations that we used in this paper.

Assume the new call arrival for both voice and video follows a Poisson process with mean arrival rates $\lambda_{1}$ and $\lambda_{2}$, respectively. Let the call holding time be exponentially distributed with mean 
$1 / \mu_{1}$ for voice and $1 / \mu_{2}$ for video. Then the traffic intensities for voice and video calls are $\rho_{1}=\lambda_{1} / \mu_{1}$ and $\rho_{2}=\lambda_{2} / \mu_{2}$. Given that there are $m$ active video calls at the time of consideration, let the probability that there are $i$ video calls after time $t$ be $P_{i}(t, m, M)$, where $M$ is the total number of video channels available. Obviously, $P_{i}(t, m, M)$ is a transient state probability of a $M / M / k$ queueing system and it can be approximated by the following equations [1]:

$$
P_{k}(t, m, M)=\frac{P_{k}(t, m, \infty)}{\sum_{l=0}^{M} P_{l}(t, m, \infty)}
$$

where

$$
\begin{aligned}
P_{k}(t, m, \infty)= & \frac{1}{k !} \exp \left[\left(1-e^{\mu_{2} t}\right) \frac{\lambda_{2}}{\mu_{2}}\right] \\
& \cdot e^{-\mu_{2} k t} \cdot\left(1-e^{-\mu_{2} t}\right)^{m} \\
& \cdot \sum_{r=0}^{\infty} \frac{1}{r !} \exp \left[\left(e^{\mu_{2} t}-1\right) \frac{\lambda_{2}}{\mu_{2}}\right]^{r} \\
& \cdot \frac{(m+r) !}{(m-k+r) !} \cdot\left(1-e^{-\mu_{2} t}\right)^{r-k}
\end{aligned}
$$

for $k \leq m$

or

$$
\begin{aligned}
P_{k}(t, m, \infty)= & \frac{1}{k !} \exp \left[\left(1-e^{\mu_{2} t}\right) \frac{\lambda_{2}}{\mu_{2}}\right] \\
& \cdot e^{-\mu_{2} k t} \sum_{r=0}^{\infty}\left[\left(e^{\mu_{2} t}-1\right) \frac{\lambda_{2}}{\mu_{2}}\right]^{k-m+r} \\
& \cdot \frac{(k+r) !}{r !(k-m+r) !} \cdot\left(1-e^{-\mu_{2} t}\right)^{r}
\end{aligned}
$$

for $k>m$.

Suppose $S$ video channels are to be simultaneously borrowed. Then without violating the video blocking requirement $P_{r}, X_{S}$ the maximum time that the $S$ video channels can be simultaneously lent is given by the solution of the following equation:

$$
P_{M-S}\left(X_{S}, m, M-S\right)=P_{r} .
$$

It is easy to verify that $X_{1}>X_{2}>\cdots>X_{M-S}=0$. If all $k$ borrowed video channels can be returned within the borrowing time $X_{k}(0<k<M-S)$, the video call blocking requirement will not be violated. Then the problem is to find out how long the video channels will be borrowed.

It is important to emphasize that for a practical system, all call arrivals are sequential because in each cell of a cellular system, there is only one base station and it needs to serve each arrived call one by one. This implies that simultaneously borrowing more than one video channels, i.e., $S>1$, will not happen. But multiple video channels can still be borrowed at different times, one by one. From now on, we only consider $S=1$ unless otherwise specified.

Immediate channel reallocation is adopted in our proposed channel borrowing strategy. It means that when a voice call holding a voice channel is finished and if there is a borrowed video channel, then the voice call on the borrowed video channel

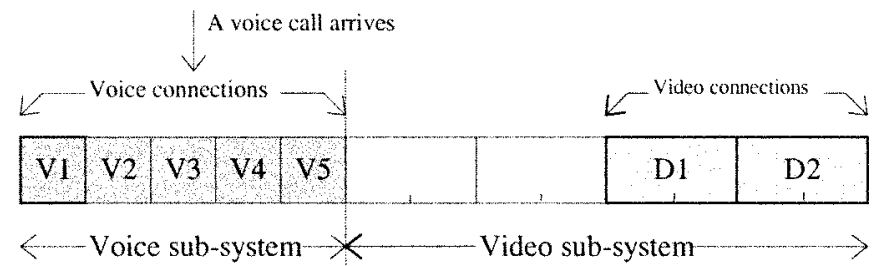

(a)

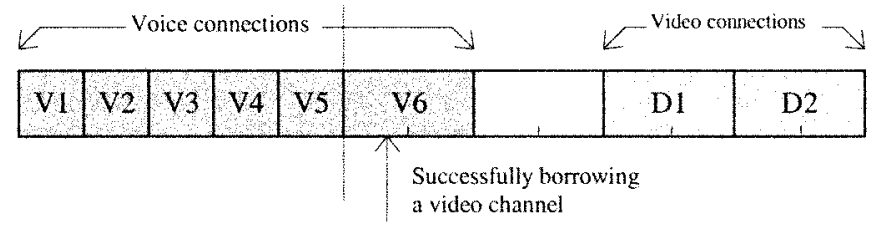

(b)

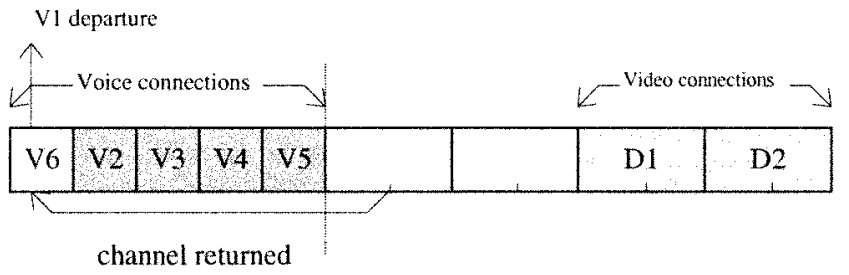

(c)

Fig. 2. Example of the proposed borrowing strategy: (a) at time $T$ : a voice new call arrives; (b) at time $T+\delta$ : borrowing successfully; and (c) borrowing channel is returned.

is immediately reallocated to the just released voice channel. The borrowed video channel is then returned. An example is shown in Fig. 2. For the sake of presentation simplicity, here we assume each borrowed video channel can only carry one voice call.

When a voice call arrives and found all $N$ voice channels are busy, it initiates a channel borrowing request. Using (2) with $S=1$, we can find out the maximum channel lending time $X_{1}$. Since the duration of each call is exponentially distributed, once the video channel is lent, there exists a probability $P_{s}$ that the lent video channel cannot be returned in time, i.e., within $X_{1}$. Let $P_{s}^{*}$ (e.g., $P_{s}^{*}=0.1$ ) be the system requirement on $P_{s}$. When a video channel borrowing request is initiated, the request will be granted only if $P_{s} \leq P_{s}^{*}$; otherwise, the request will be rejected. A suitable set of $P_{s}^{*}$ values can keep the video call blocking probability as close to its QoS requirement as possible. Numerical examples will be constructed in Section VI-A for studying the effect of different $P_{s}^{*}$ values on the call blocking probability.

\section{ANALYTICAL MODEL}

In this section, an analytical model for a simplified system where each video channel can carry a single voice call is constructed. Let $P_{B 1}$ and $P_{B 2}$ be the call blocking probabilities of voice and video when the borrowing strategy is used. Similarly let $P_{N}$ and $P_{M}$ be the call blocking probabilities of voice and video traffic without using the borrowing strategy. The values of $P_{N}$ and $P_{M}$ can be obtained using the Erlang-B formula. Throughout the paper, $P_{N}$ and $P_{M}$ are used for comparing the effect of using channel borrowing. 

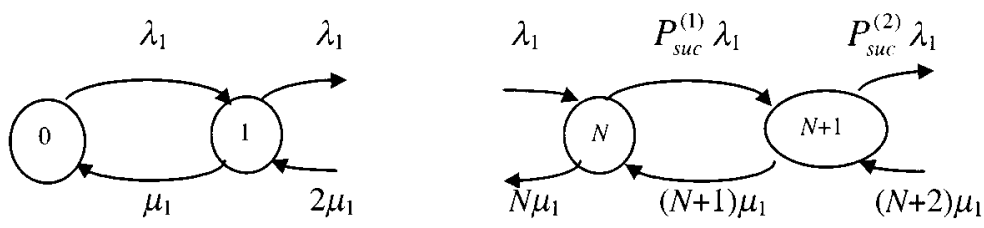

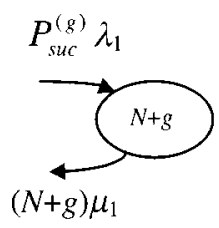

Fig. 3. The channel occupancy of voice calls.

\section{A. Derivation of Voice Call Blocking Probability}

Let $P_{\text {suc }}^{(g)}$ be the probability of a successful video channel borrowing given that $(g-1)$ video channels have already been borrowed by voice calls. Let us first consider the simplest case of $P_{\text {suc }}^{(1)}$, the probability of successful borrowing the first video channel. When a voice call arrives and found all $N$ voice channels and $m$ video channels are busy, $X_{1}$ the maximum time that a video channel can be borrowed without violating the video call blocking requirement $P_{r}$ can be found from (2). Then $P_{s}$ the probability that no voice call will be finished within time period $X_{1}$ is given by

$$
P_{s}=e^{-(N+1) \mu_{1} X_{1}} .
$$

We must have $P_{s} \leq P_{s}^{*}$ for a video channel to be borrowed. Substituting (3) into this inequality and after rearrangement, we have the minimum channel borrowing time $\theta$, where

$$
\theta=-\frac{\ln P_{s}^{*}}{\mu_{1}(N+1)} \leq X_{1} \text {. }
$$

This means that for a video channel to be successfully borrowed (without violating $P_{r}$ and $P_{s}^{*}$ ), the maximum video channel lending time $X_{1}$ must be greater than the minimum channel borrowing time $\theta$. Then conditioning on the video channel occupancy, the probability that the video channel borrowing is successful is given by

$$
P_{\mathrm{suC}}^{(1)}=\sum_{i=0}^{M-1} P_{i} \cdot P\left\{X_{i} \geq \theta \mid P_{M-1}\left(X_{1}, i, M-1\right)=P_{r}\right\}
$$

where $P_{i}=\left(\rho_{2}^{i} / i !\right) \cdot\left(\sum_{k=0}^{M} \rho_{2}^{k} / k !\right)^{-1}$ is the probability that $i$ video channels are busy.

Next, we consider $P_{\text {suc }}^{(g)}$. When a voice call arrives and found all $N$ voice channels and $(m+g-1)$ video channels are busy (where $(g-1)$ video channels are carrying voice calls), the maximum time that the $g$-th video channel can be borrowed without violating the video call blocking requirement is very difficult to find. To simplify the analysis, we approximate this maximum borrowing time by considering a system consists of $N$ voice calls and $(M-g+1)$ video channels (instead of $M$ video channels).

Then, when the new voice call arrives, the maximum channel borrowing time is approximated by the maximum time that the first video channel can be borrowed from a system with $(M-$ $g+1)$ video channels, where $m$ out of $(M-g+1)$ video channels are busy. Again, let $X_{1}$ be the maximum time that a video channel can be borrowed without violating the video call blocking requirement $P_{r}$. It can be found from (2) by substituting $M$ with $(M-g+1)$ and $S=1$. Then $P_{s}$, the probability that no voice call will be finished within time period $X_{1}$, and $\theta$, the minimum channel borrowing time, are given by (3) and (4), respectively.

Similar to (5), the probability that the $g$-th video channel borrowing is successful (given that $(g-1)$ video channels have been borrowed) can be found

$$
\begin{gathered}
P_{\text {suc }}^{(g)}=\sum_{i=0}^{M-g} P_{i} \cdot P\left\{X_{1} \geq \theta \mid P_{M-g+1}\right. \\
\left.\left(X_{1}, i, M-g+1\right)=P_{r}\right\}
\end{gathered}
$$

where $P_{i}=\left(\rho_{2}^{i} / i !\right) \cdot\left(\sum_{k=0}^{M} \rho_{2}^{k} / k !\right)^{-1}$ is again the probability that $i$ video channels are busy.

Therefore, the voice call blocking probability $\left(P_{B 1}\right)$ using channel borrowing can be expressed as

$$
P_{B 1}=\sum_{i=1}^{g}\left(1-P_{\text {suc }}^{(i)}\right) P_{N+i-1}+P_{N+g}
$$

where $P_{\text {suc }}^{(i)}$ is the probability that the $i$-th video channel can be successfully borrowed, and $P_{j}$ is the steady state probabilities of $j$ voice calls in the system described by the $(N+g+1)$-state diagram shown in Fig. 3. Solving the above Markovian chain, we can get the state probability of $i$ voice calls:

$$
P_{i}=\left(\rho_{1}^{i} / i !\right)\left\{\sum_{k=0}^{N} \frac{\rho^{k}}{k !}+\sum_{j=1}^{g} \frac{\left(P_{\mathrm{suc}}^{(1)} P_{\mathrm{suc}}^{(2)} \cdots P_{\mathrm{suc}}^{(j)}\right) \rho_{1}^{(N+j)}}{(N+j) !}\right\}^{\text {for } i=0,1,2, \ldots, N}
$$

$$
\begin{aligned}
P_{i}= & \frac{\left(P_{\mathrm{suc}}^{(1)} P_{\mathrm{suc}}^{(2)} \cdots P_{\mathrm{suc}}^{(i-N)}\right) \rho_{1}^{i}}{i !} \\
& \times\left\{\sum_{k=0}^{N} \frac{\rho^{k}}{k !}+\sum_{\substack{j=1 \\
\text { for } i=N+1, N+2, \ldots, N+g}}^{g}\right\}^{(9)}
\end{aligned}
$$

where $\rho_{1}$ is the voice traffic intensity.

\section{B. Derivation of Video Call Blocking Probability}

Next, we derive the video call blocking probability $P_{B 2}$. The two-dimensional Markovian chain shown in Fig. 4 shows the channel occupancy of the video calls, where state $(i, j)$ denotes that the system has $i$ active video calls and $j$ active voice calls occupying $j$ borrowed video channels. Suppose when there are 

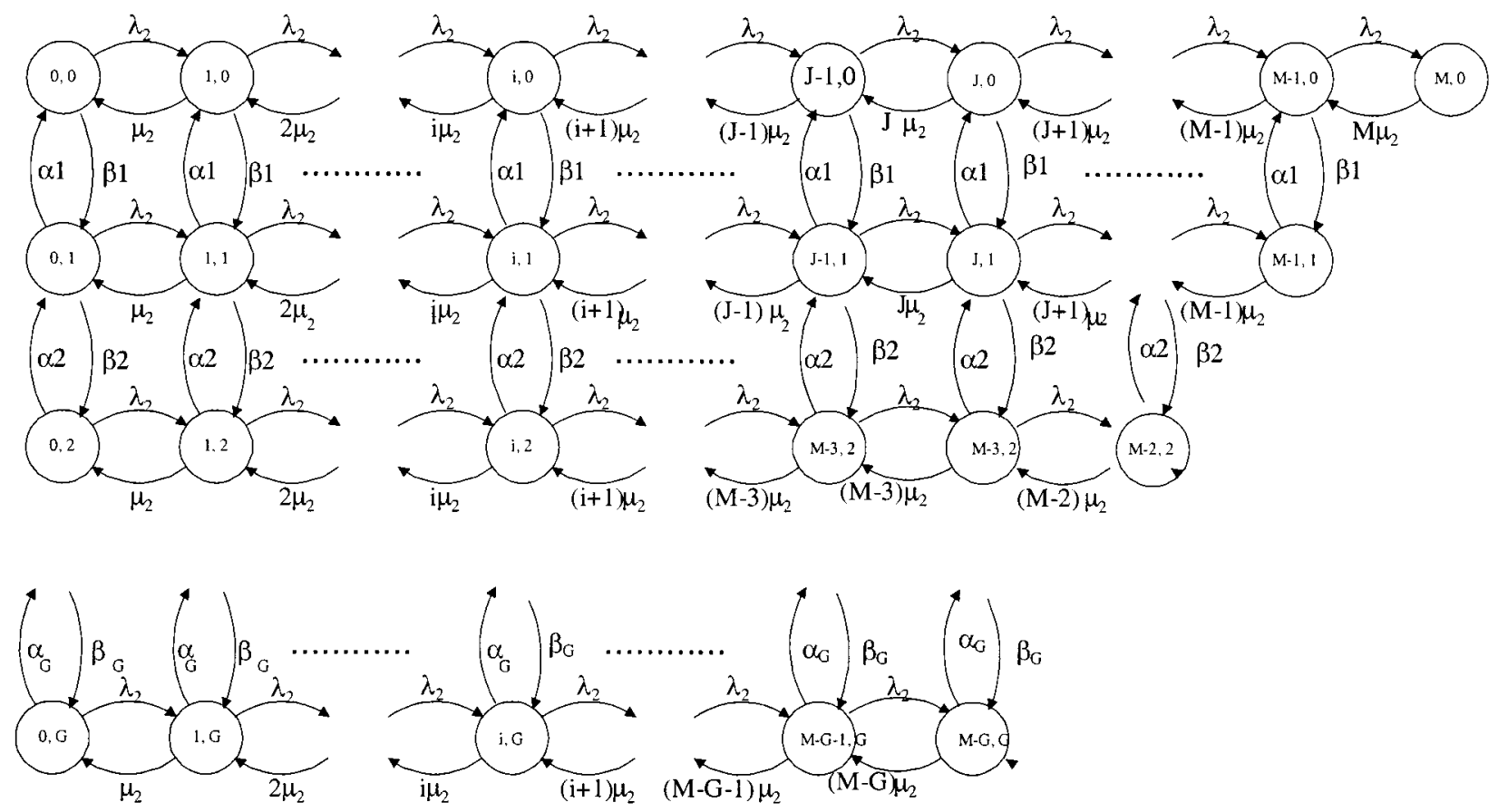

Fig. 4. The channel occupancy of video calls.

$x$ video channels are lent to voice calls and $J_{x}$ or more video calls in the system, no video channel can be further borrowed by voice calls. The value of $J_{x}$ can be obtained from (2)-(4) in the previous section.

From Fig. 4, the following set of balancing equations can be obtained:

$$
\left.\begin{array}{l}
\alpha_{1} P_{0,1}+\mu_{2} P_{1,0}-\left(\lambda_{2}+\beta_{1}\right) \cdot P_{0,0}=0 \\
\lambda_{2} P_{i-1,0}+(i+1) \mu_{2} P_{i+1,0}+\alpha_{1} P_{i, 1}-\left(i \mu_{2}+\lambda_{2}+\beta_{1}\right) \\
\quad \cdot P_{i, 0}=0 \text { for } 0<i<M \\
\lambda_{2} P_{M-1,0}-M \mu_{2} \cdot P_{M, 0}=0 \\
\quad \beta_{j} P_{0, j-1}+\mu_{2} P_{1, j}+\alpha_{j+1} P_{0, j+1}-\left(\lambda_{2}+\alpha_{j}+\beta_{j+1}\right) \\
\quad \cdot P_{0, j}=0 \text { for } 0<j<G \\
\lambda_{2} P_{i-1, j}+(i+1) \mu_{2} P_{i+1, j}+\beta_{j} P_{i, j-1}+\alpha_{j+1} P_{i, j+1} \\
\quad-\left(i \mu_{2}+\lambda_{2}+\alpha_{j}+\beta_{j+1}\right) \cdot P_{i, j}=0 \\
\quad \text { for } 0<i<M-j ; \quad 0<j<G \\
\lambda_{2} P_{M-j-1, j}+\beta_{j} P_{M-j, j-1}-\left((M-j) \mu_{2}+\alpha_{j}\right) P_{M-j, j} \\
\quad=0 \text { for } 0<j<G \\
\beta_{G} P_{0, G-1}+\mu_{2} P_{1, G}-\left(\lambda_{2}+\alpha_{G}\right) \cdot P_{0, G}=0 \\
\lambda_{2} P_{i-1, G}+(i+1) \mu_{2} P_{i+1, G}+\beta_{G} P_{i, G-1} \\
\quad-\left(i \mu_{2}+\lambda_{2}+\alpha_{G}\right) \cdot P_{i, G}=0 \quad \text { for } 0<i<M-G \\
\lambda_{2} P_{M-G-1, G}+\beta_{G} P_{M-G, G-1} \\
\quad-\left((M-j) \mu_{2}+\alpha_{G}\right) P_{M-G, G}=0 \\
\quad \text { for } i=0,1, \ldots, M ; \quad j=0,1, \ldots, G
\end{array}\right\}
$$

where $\alpha_{j}$ is the departure rate of the voice call holding the borrowed video channel, $G$ is the maximum video channels that can be borrowed, and $\beta_{j}$ is the voice call arrival rate to a borrowed video channel. $\beta_{j}$ is given by

$$
\beta_{j}=P_{N+j} \lambda_{1}
$$

where

$$
\begin{aligned}
P_{N+j}= & \frac{\left(P_{\mathrm{suc}}^{(1)} P_{\mathrm{suc}}^{(2)} \cdots P_{\mathrm{suc}}^{(j)}\right) \rho_{1}^{(N+j)}}{(N+j) !} \\
& \times\left\{\sum_{k=0}^{N} \frac{\rho^{k}}{k !}+\sum_{i=1}^{g} \frac{\left(P_{\mathrm{suc}}^{(1)} P_{\mathrm{suc}}^{(2)} \cdots P_{\mathrm{suc}}^{(i)}\right) \rho_{1}^{(N+i)}}{(N+i) !}\right\}^{-1}
\end{aligned}
$$

is obtained from (9). It is the probability that all $(N+j)$ voice channels (including $j$ channels borrowed from video) are busy. Note that we have assumed that the overflow traffic $\beta_{j}$ follows a Poisson process.

$\alpha_{j}$, the departure rate of the voice call holding the borrowed video channel, can be approximated by

$$
\alpha_{j}=(N+j) \cdot \mu_{1}
$$

It should be noted that using the above equation, we have assumed that all borrowed video channels are indistinguishable and the immediate channel reallocation policy shown in Fig. 2 is used. 


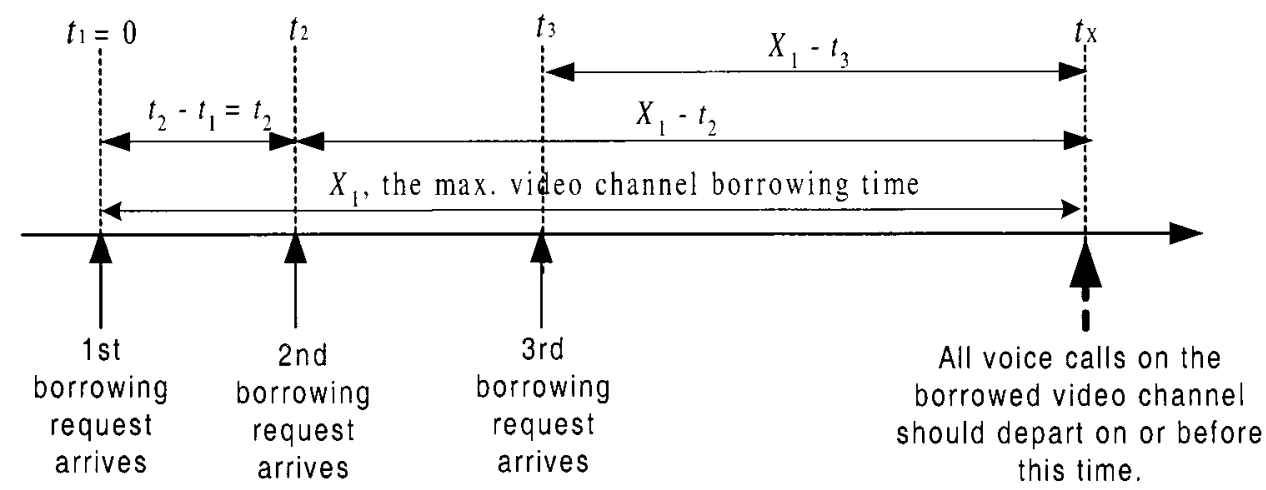

Fig. 5. Timing diagram for borrowing a video channel to carry multiple voice calls. Call departure is not shown. $t_{x}-t_{1}=X_{1} ; t_{x}-t_{2}=X_{1}-t_{2} ; t_{x}-t_{3}=$ $X_{1}-t_{3}$.

Solving (10)-(12), the video call blocking probability $P_{B 2}$ is the summation of all the blocking state probabilities in Fig. 4, or

$$
P_{B 2}=\sum_{i=0}^{M} P_{M-i, i}
$$

\section{Borrowing a Video Channel for Multiple Voice CALLS}

We relax the constraint that each video channel can only carry a single voice call in this section. We consider a general system that can borrow one video channel for carrying up to $n$ voice calls, where $n>1$. In this case, we cannot find the analytical expressions for the voice and video call blocking probabilities because the number of state variables involved is too large to be handled. In the following, a heuristic channel borrowing rule is designed and its performance is evaluated in Section VI by simulations.

Fig. 5 shows the timing diagram for borrowing a video channel to carry multiple voice calls. When a voice call arrives at $t_{1}=0$ and found all voice channels are busy, the first borrowing request for a video channel is initiated. Based on the current video channel occupancy $m$, the maximum video channel borrowing time without violating the video call QoS requirement $X_{1}$ is found by solving (2) with $S=1$. If the expected channel borrowing time $\theta$ is less than $X_{1}$, the borrowing request is granted and the call is assigned to the first time slot of the borrowed video channel. We refer it as the first voice call. Suppose the first voice call is not finished when the second voice call arrives, it initiates the second channel borrowing request at $t_{2}$. We check if the remaining video channel borrowing time $X_{1}-t_{1}$ is sufficient for both calls, the first call and the second call (if admitted), to be finished by $t_{x}$. Since the holding time is assumed to be exponentially distributed, it is memoryless. Thus at time $t_{2}$, we consider the remaining call duration for the first call and the call duration for the second call (under consideration), follow the same negative exponential distribution with mean $1 / \mu_{1}$. To check if the second call should be assigned to the second time slot of the borrowed video channel, two cases need to be considered. First, both calls cannot be completed by $t_{x}$. Second, one of the two calls cannot be completed by $t_{x}$. If the total probability for these two cases is less than $P_{s}^{*}$, the pre-defined requirement on the probability that the borrowed video channel cannot be returned within $X_{1}$, the second call borrowing request is granted. Otherwise, the second call is rejected.

Similarly, suppose all the previous $q-1$ calls are still ongoing and holding the time slots on the borrowed video channel when the $q$-th call arrives at $t_{q}$. The $q$-th call request is granted if the total probability of at least one call is not finished by $t_{x}$ is less then $P_{s}^{*}$. Otherwise, the $q$-th call is rejected. It is obvious that $q \leq n$ and $X_{1}-t_{q}>0$. The above channel borrowing policy can be summarized by the following inequality:

$$
\sum_{p=0}^{q-1} r_{q}^{(N+q)-p} \prod_{i=0}^{P-1}\left(1-r_{q}^{N+q-i}\right) \leq P_{s}^{*}
$$

where $r_{q}=e^{-\mu_{1}\left(x_{1}-t_{q}\right)}$, and $N$ is the total number of voice channels. The left-hand side of the above inequality is the total/summation of the probabilities that at least one call out of $q$ calls (i.e., $q-1$ ongoing calls plus and one call under consideration) can not be completed by $t_{x}$. Inside the summation sign, the first term $r_{q}^{(N+q)-p}$ is the probability that $(q-p)$ calls cannot be finished by $t_{x}$. The second term $\prod_{i=0}^{P-1}\left(1-r_{q}^{N+q-i}\right)$ is the probability that $p$ calls have been finished by $t_{x}$.

For a more general system that multiple video channels can be borrowed and on each borrowed video channel, multiple voice calls can be carried, the channel borrowing rule is very difficult to design and is beyond our ability at this moment. We argue that for a practical system that each video channel can support multiple voice calls, borrowing of more than one video channels is seldom. This is because 1) borrowing one video channel is borrowing of multiple voice channels; and 2) channel borrowing is only used to solve the temporary traffic congestion; if such events persist, the bandwidth/time slots should be re-partitioned for giving more time slots to voice.

\section{CHANNEl Borrowing FOR A PRACTICAL SYSTEM}

Let $m$ denote the number of currently active video calls (i.e., video channel occupancy). Given the number of video channels that have already been lent to voice calls is $x, J_{x}$ denote the threshold on $m$ such that if $m<J_{x}$, further video channel borrowing is allowed; otherwise, no more video channel can be borrowed. Refer to Section III-B and Fig. $4, J_{x}$ is different for different values of $m$ and $x$. Using (2)-(4), $J_{x}$ is obtained for various values of $(m, x)$ pair. (If multiple voice calls per 
TABLE II

SyStEM PARAMETERS For ANALYSIS AND SimULATION

\begin{tabular}{l|c|l}
\hline \multicolumn{1}{c|}{ Parameters } & Symbols & \multicolumn{1}{c}{ Values } \\
\hline Voice arrival rate & $\lambda_{1}$ & varied \\
Voice departure rate & $\mu_{1}$ & $1 / 3$ calls $/ \mathrm{min}$. \\
Video arrival rate & $\lambda_{2}$ & 1.5 calls $/ \mathrm{min}$. \\
Video departure rate & $\mu_{2}$ & $1 / 3$ calls $/ \mathrm{min}$. \\
Number of voice channels & $N$ & 20 \\
Number of video channels & $M$ & 10 \\
Video blocking requirement & $P_{r}$ & 0.01 \\
System requirement on the prob. of no voice call leaving & $P_{s}{ }^{*}$ & varied \\
the system within time $X_{1}$ & & \\
\hline
\end{tabular}

video channel is allowed, (14) is also used and the resulting $J_{x}$ depends on $(m, x)$ pair as well as the number of idle slots in the already borrowed video channels.) Then the resulting values of $J_{x}$ are stored in a table called channel borrowing table.

For a practical wireless system, the channel borrowing table is pre-calculated and then loaded into the system memory at the base station. Thus no real-time calculation of the lookup table is required. For a system that supports single voice call per video channel, when a new voice call arrives and found all $N$ voice channels are busy, a table-lookup is performed to check if a video channel can be borrowed based on the current video channel occupancy and the number of video channels that have already been lent, i.e., $(m, x)$. If $m<J_{x}$, a new video channel is borrowed to carry the arrived voice call; otherwise, the call is blocked.

For a system that supports multiple voice calls per video channel, one more index entry is added in the channel borrowing table: the number of idle slots in the borrowed video channel. In this case, when a voice call arrives and found all voice channels are busy, the system checks if any video channels have already been borrowed. Assume $g$ video channels have been borrowed. Check the last borrowed video channel (i.e., channel $g$ ) for possible idle time slots. Perform a table-lookup and check if the available number of idle slots in video channel $g$ is sufficient to carry the current call. If yes, the call is assigned to the idle slot; if not, try to borrow a new idle video channel by performing another table-lookup (similar to the system that supports single voice call per video channel). It should be noticed that in this paper, we only designed the channel borrowing rule, as summarized by (14), for borrowing one single video channel for carrying multiple voice calls. The borrowing of multiple video channels is beyond our scope.

When a voice call holding a voice channel is finished, according to the order of video channel borrowing, the first voice call on the borrowed video channel is re-assigned to the just released voice channel. When there is no borrowed video channel or a call holding a video slot/channel is finished, no channel re-assignment is allowed/needed.

\section{NUMERICAL AND SimUlation Results}

The performance of the proposed resource sharing strategy is evaluated in this section. First we investigate the simple case of borrowing one video channel for one voice call in details. The effect on choosing different values of $P_{s}^{*}$ is investigated.

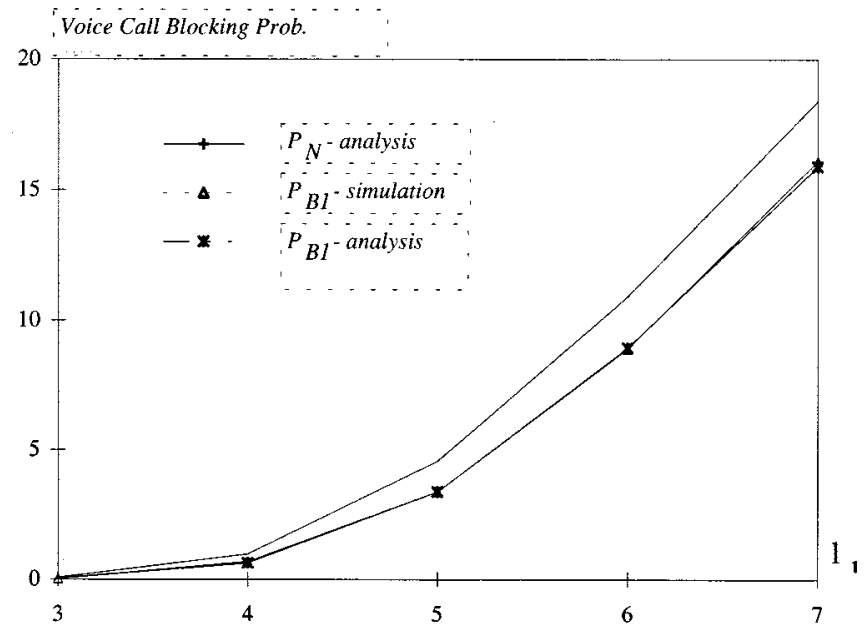

Fig. 6. Voice call blocking prob. versus voice call arrival rate $\lambda_{1}: P_{s}^{*}=$ $0.1, P_{r}=0.01$

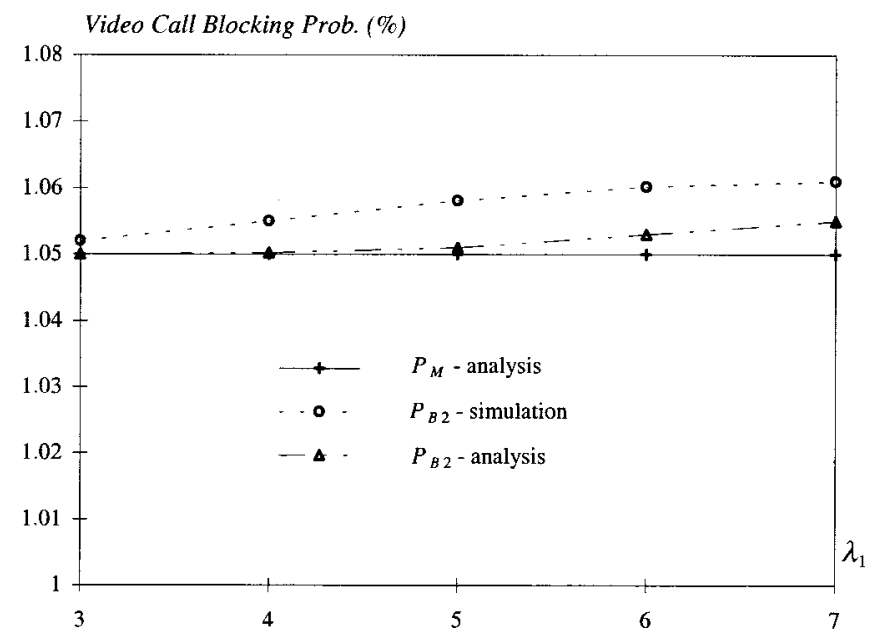

Fig. 7. Video call blocking prob. versus voice call arrival rate $\lambda_{1}: P_{s}^{*}=$ $0.1, P_{r}=0.01$

Then we focus on multiple channel borrowing scenario in which each video channel can carry one voice call. Both analytical and simulation results are obtained and compared. Finally, we study the effect of borrowing one video channel for carrying multiple voice calls using simulations. Table II summarizes some useful parameters. 


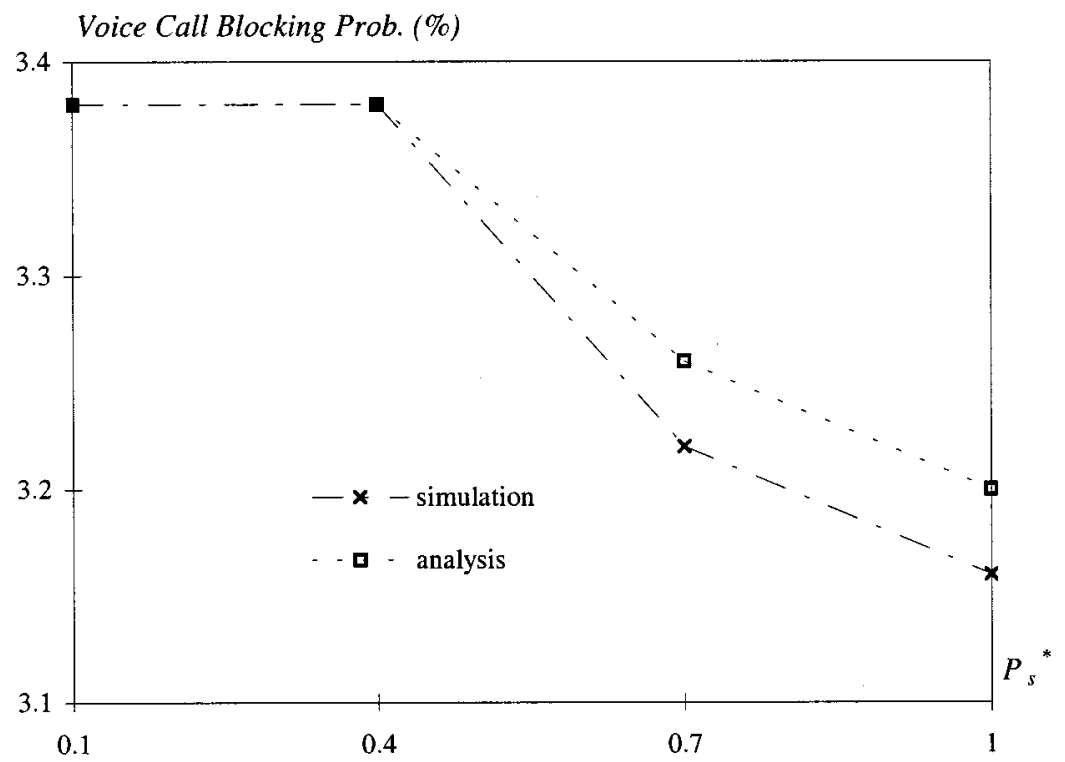

Fig. 8. Voice call blocking prob. versus $P_{s}^{*}: \lambda_{1}=5$ calls $/ \mathrm{min} ., P_{r}=0.01$.

Video Call Blocking Prob. (\%)

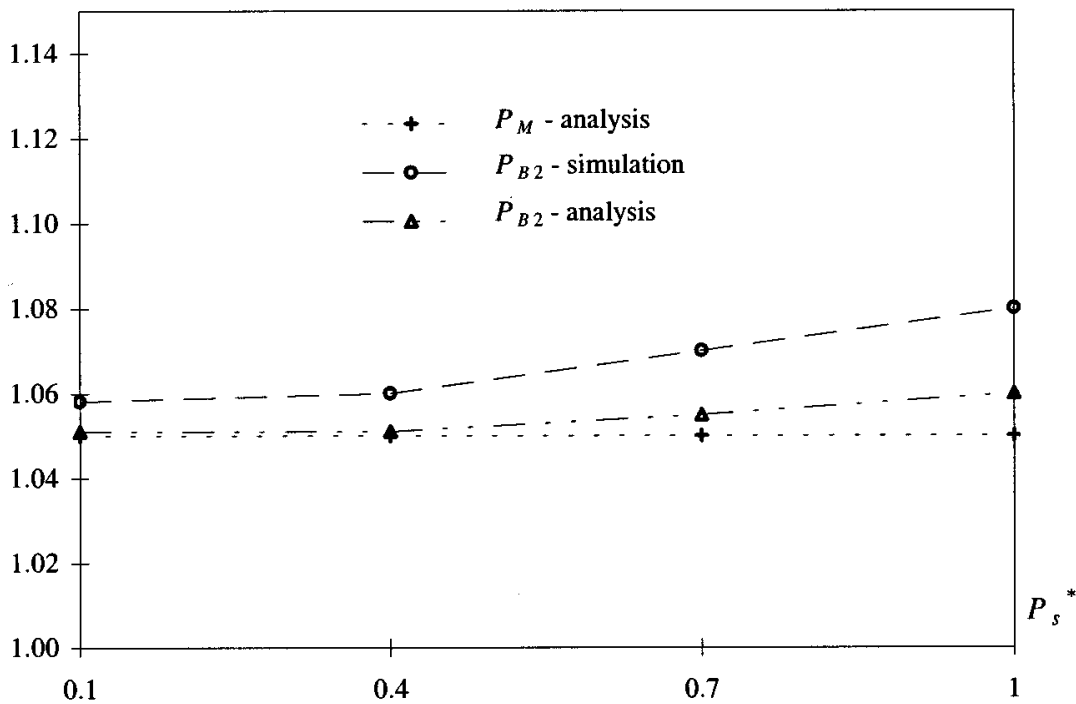

Fig. 9. Video call blocking prob. versus $P_{s}^{*}: \lambda_{1}=5$ calls $/ \mathrm{min} ., P_{r}=0.01$.

\section{A. Borrowing a Single Video Channel for a Single Voice Call}

First, we consider the case that only one video channel can be borrowed and the borrowed video channel can only carry a single voice call. Figs. 6 and 7 show the blocking probabilities of voice and video versus voice call arrival rate. The probability requirement on no voice call finished during the video channel borrowing time is set to $P_{s}^{*}=0.1$. The video call blocking probability requirement is fixed at $P_{r}=0.01$. The video call arrival rate is fixed at 1.5 calls $/ \mathrm{min}$ such that without using channel borrowing strategy, the video blocking of $P_{M}=0.0105$ is obtained using Erlang-B formula. We consider 0.0105 is close enough to $P_{r}=0.01$ and thus satisfied the video blocking requirement.

From Fig. 6 we can see that the voice call blocking probability decreases when channel borrowing strategy is used and the reduction in voice call blocking probability increases as voice call arrival rate increases. At $\lambda_{1}=7$ calls $/ \mathrm{min}$, the reduction in voice call blocking probability is 0.024 (i.e., $P_{B 1}-P_{N}$ ). From Fig. 7 we can find that the corresponding increase in video call blocking probability is insignificant and in the worst case, i.e., $\lambda_{1}=7$ calls $/ \mathrm{min}, P_{B 2}=0.0106$ is only increased by about 0.0001 as compared to $P_{M}=0.0105$. Furthermore, we can see that the analytical results of $P_{B 1}$ and $P_{B 2}$ shown in both figures match the simulation results very well.

Then we study the effect of changing $P_{s}^{*}$, the system requirement on the probability that no voice call is finished within the channel borrowing time $X_{1}$. Voice call arrival rate is fixed at $\lambda_{1}=5$ calls $/ \mathrm{min}$. Figs. 8 and 9 show voice $\left(P_{B 1}\right)$ and video $\left(P_{B 2}\right)$ call blocking probabilities versus $P_{s}^{*}$, where $P_{s}^{*}$ varies from 0.1 to 1.0 . From (4) we found $\theta=0.329,0.131,0.051$, and $0 \mathrm{~min}$, for $P_{s}^{*}=0.1,0.4,0.7$, 


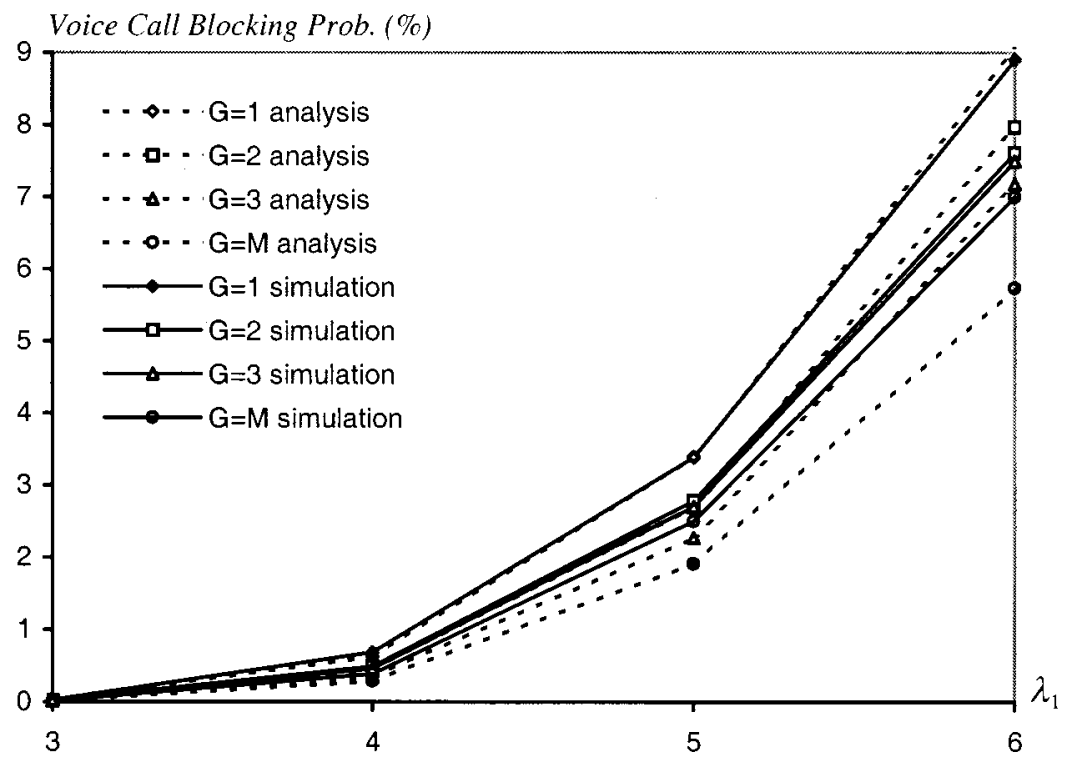

Fig. 10. Voice call blocking prob. versus $\lambda_{1}: P_{s}^{*}=0.1, P_{r}=0.01, S=0,1,2,3, M$.

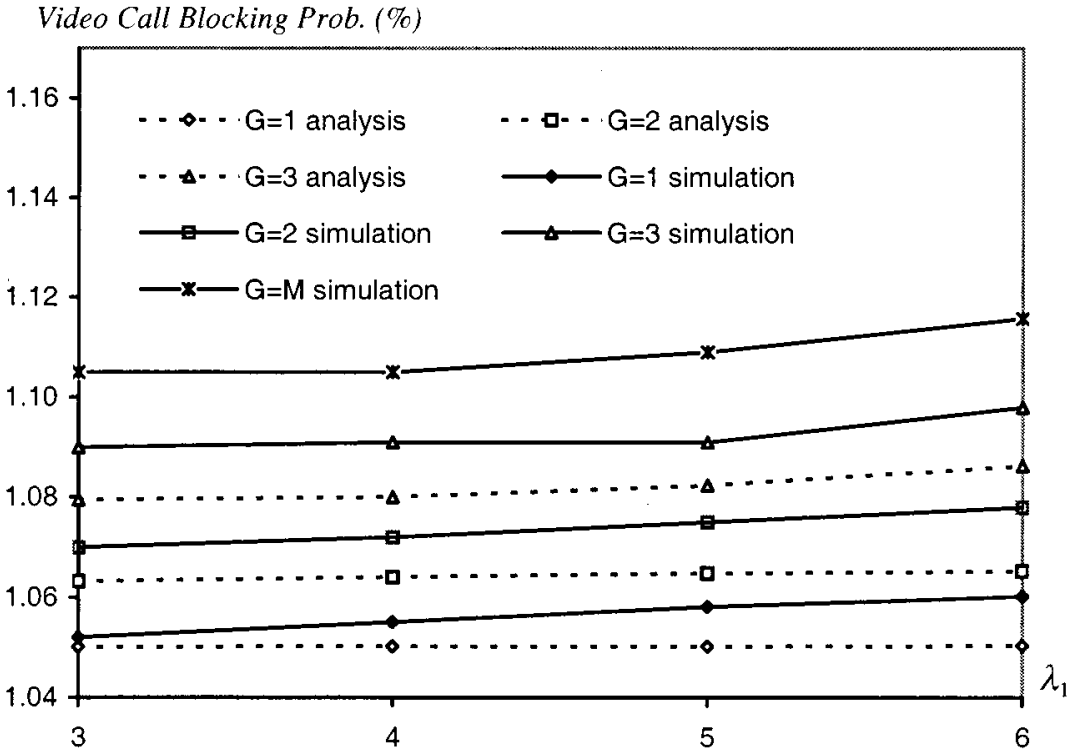

Fig. 11. Video call blocking prob. versus $\lambda_{1}: P_{s}^{*}=0.1, P_{r}=0.01, S=0,1,2,3, M$.

and 1.0, respectively. From (1) and (2), we have $X_{1}=5.508$, $4.829,4.009,3.031,1.970,1.049,0.440,0.120,0.001$, and 0 $\min$, for $m$ the number of active video calls varies from 0 to 9 . Again, from (4), we can get the value for parameter $J_{x}$ (refer to Fig. 4). When the system has $J_{x}$ or more active video calls, no further video channel can be lent to voice calls. We have $J_{x}=7,7,8$ and 9 for $P_{s}^{*}=0.1,0.4,0.7$, and 1.0 , respectively.

From Figs. 8 and 9, we can see that voice call blocking $P_{B 1}$ and the video call blocking $P_{B 2}$ remain unchanged for $P_{s}^{*} \leq$ 0.4 . This is because the values of $J_{x}$ are the same when $P_{s}^{*}$ is set to 0.1 and 0.4 . When $P_{s}^{*}>0.4$, the voice blocking probability drops quickly and the QoS requirement on video blocking $P_{r}$ is slightly increased. Hence, we can conclude that the video QoS can be guaranteed using channel borrowing strategy when a suitable set of $P_{s}^{*}$ values is used. In general, the smaller the value of $P_{s}^{*}$, the more difficult to borrow a video channel and the probability of violating $P_{r}$ is lower. The vice versa is true for a large value of $P_{s}^{*}$. The optimal value of $P_{s}^{*}$ thus depends on the tolerance of a system on the probability of violating its video QoS.

\section{B. Borrowing Multiple Video Channels for Multiple Voice Calls}

Next, we consider the multiple channel borrowing scenario. Again, we assume each video channel can only carry one voice call. Figs. 10 and 11 show the blocking probabilities of voice and video versus voice arrival rate. Let $G$ be the maximum number of video channels available for lending to voice calls, where $G \leq M$. Results for $G=1,2,3$ and $M$ are shown in the figures. For the case of $G=M$, that means all video channels can 


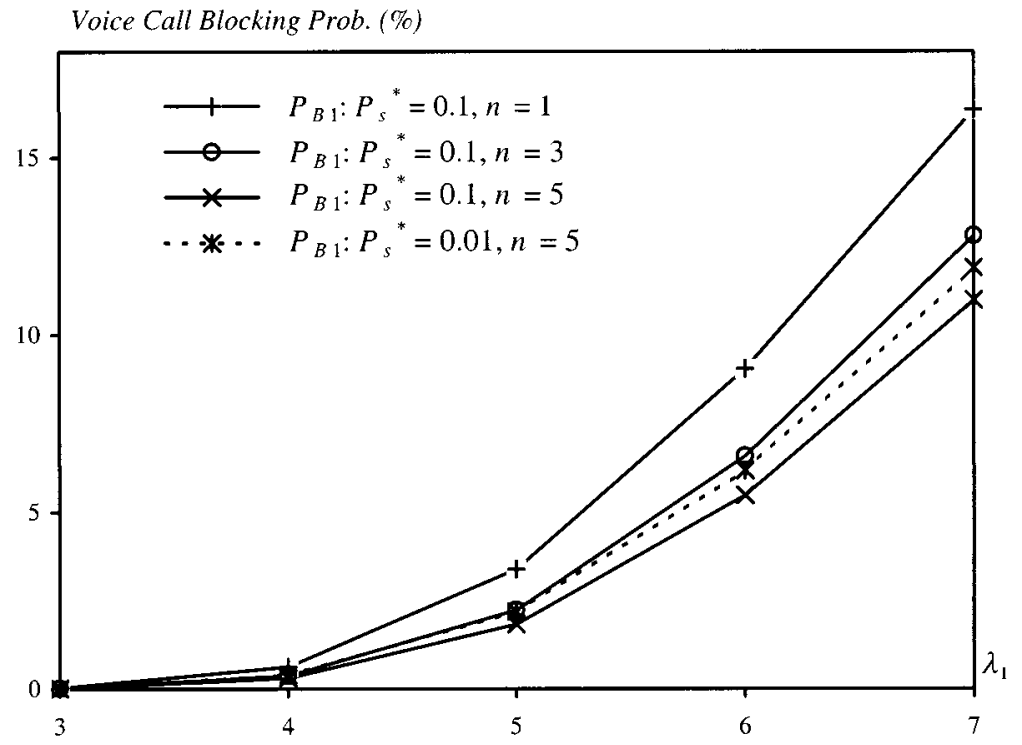

Fig. 12. Voice call blocking prob. versus $\lambda_{1}$ for different values of $P_{s}^{*}$ and $n . P_{r}=0.01$.

be borrowed by voice calls. The probability requirement on no voice call is finished during the video channel borrowing time is set to $P_{s}^{*}=0.1$, and the video blocking probability requirement is fixed at $P_{r}=0.01$.

From Fig. 10 we can see that, compared to one channel borrowing strategy in Fig. 6, the voice call blocking probability is further reduced when multiple channel borrowing strategy is used. As expected, the larger the value of $G$, the lower the voice call blocking probability we can get. When $G=M$, at $\lambda_{1}=6$ calls $/ \mathrm{min}$, the reduction in voice call blocking probability is about 0.02 (based on simulation results) as compared to the case with $G=1$. From Fig. 11, we find that the corresponding increase in video call blocking probability is insignificant. Even in the worst case, i.e., $G=M$ and $\lambda_{1}=6$ calls $/ \mathrm{min}$, video call blocking $P_{B 2}=0.0112$ is only increased by about 0.0007 as compared to $P_{M}=0.0105$. We can also see that the analytical results in both figures match the simulation results very well. (Note that the analytical results for $G=M$ is not plotted because of the high computational time involved.) We can conclude that the proposed multiple channel borrowing strategy is very effective in reducing the voice call blocking probability while keeping the violation of video call blocking QoS requirement minimum.

\section{Borrowing One Video Channel for Multiple Voice Calls}

Next, we use simulations to study the effect of borrowing one video channel for carrying multiple number of voice calls using the channel borrowing policy summarized in (14). Figs. 12 and 13 show voice $\left(P_{B 1}\right)$ and video $\left(P_{B 2}\right)$ call blocking probabilities versus voice call arrival rate $\lambda_{1}$. The video call blocking QoS requirement is still set to $P_{r}=0.01$. Let the number of voice channels that can be supported by a video channel be $n$. The results of $n=1,3$ and 5 are shown in Figs. 12 and 13. The video call arrival rate remains at $\lambda_{2}=1.5$ calls $/ \mathrm{min}$ such that without using channel borrowing strategy, a video blocking of $P_{M}=0.0105$ can be obtained. From Fig. 12, we can see that when $\lambda_{1}=7$ calls $/ \mathrm{min}$ and $P_{s}^{*}=0.1$, the voice blocking prob- ability $P_{B 1}$ for $n=1,3$, and 5 are $0.1636,0.1279$, and 0.1098 , respectively. A reduction of about 0.036 and 0.054 blocking probabilities can be obtained using $n=3$ and 5 . The corresponding video call blocking probabilities have no significant changes and with values $0.01061,0.01074$, and 0.01068 , respectively.

For $P_{s}^{*}=0.01$ and $n=5$, the results are also plotted in Figs. 12 and 13 . The smaller the value of $P_{s}^{*}$, the more difficult to successfully borrow a channel. Therefore the voice call blocking probability is higher than that with $P_{s}^{*}=0.1$. On the other hand, the probability of violating video blocking requirement $P_{r}$ is reduced as indicated by the smaller values of $P_{B 2}$ in Fig. 13 .

\section{Effect of Borrowing on Radio Channel Utilization}

Next, we study the effect of channel borrowing on radio channel utilization. We define the radio channel utilization, $E$, as

$$
E=\frac{B_{a}+5 B_{\nu}}{\text { Bandwidth }}
$$

where Bandwidth $=N+5 M$. Recall that $N$ is the number of voice channels pre-allocated to voice and $M$ is the number of video channels pre-allocated to video, where each video channel occupies 5 time slots and each voice channel occupies one time slot. $B_{a}$ is the average number of the busy voice channels and $B_{v}$ is the average number of the busy video channels. Both $B_{a}$ and $B_{v}$ are obtained from simulations.

Fig. 14 shows the radio channel utilization against the voice call arrival rate. In our simulations, we keep $P_{s}^{*}=0.1$ and $P_{r}=0.01$. For video traffic, we use $\mu_{1}=1 / 3$ calls per minute and $\lambda_{1}=1.5$ calls per minute. For voice, $\mu_{2}=1 / 3$ calls per minute. From Fig. 14, we can see that without using channel borrowing, the channel utilization is the lowest. Let each borrowed video channel be used to carry one voice call, i.e., $n=1$. As the maximum number of video channels allowed for lending increases from $G=1$ to $M$, the radio channel utilization increases. Then we fix $G=1$ and vary $n$, the number of voice 


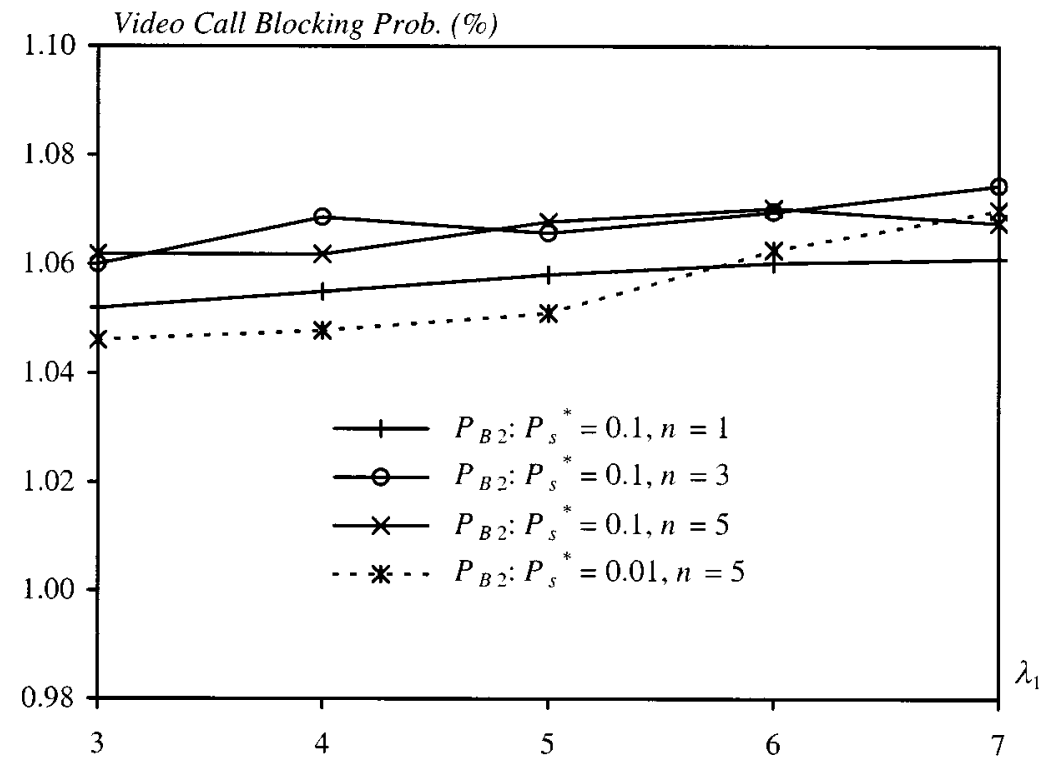

Fig. 13. Video call blocking prob. versus $\lambda_{1}$ for different values of $P_{s}^{*}$ and $n . P_{r}=0.01$.

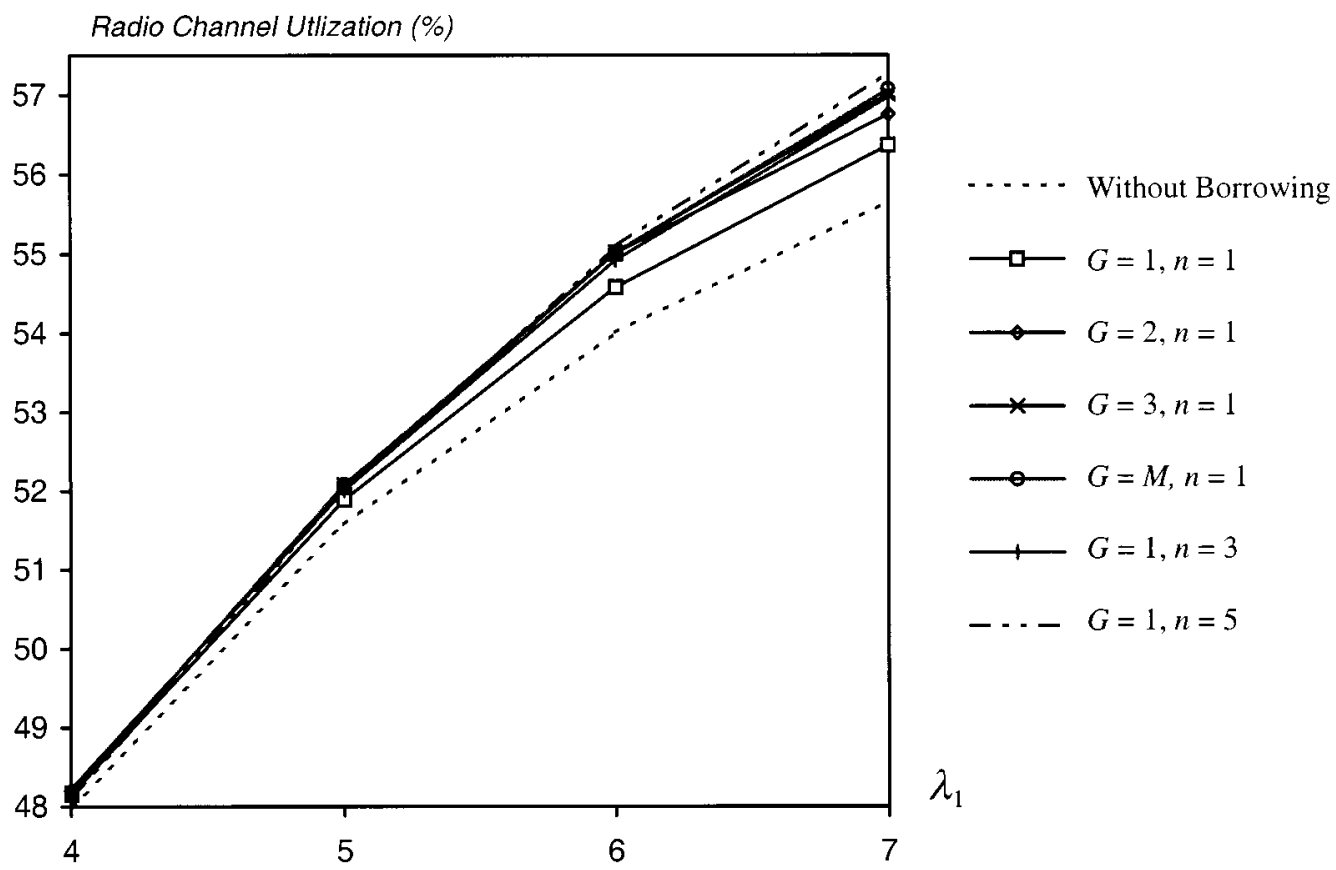

Fig. 14. Radio channel utilization versus $\lambda_{1}$ for different channel borrowing policies; $P_{s}^{*}=0.1$ and $P_{r}=0.01$.

calls per video channel. As $n$ increases, the channel utilization also increases.

Since the video call arrival rate and the video call blocking probability are fixed in our simulations, the channel utilization due to video traffic remains unchanged as 1) the voice call arrival rate increases, and 2) as different channel borrowing policies are used. For a fixed voice call arrival rate and with different channel borrowing policies, the increase in overall channel utilization is therefore due to more voice calls have been carried by the borrowed video channels. This is consistent with our earlier results that the voice call blocking probability is reduced when channel borrowing is allowed.

\section{CONCLUSION}

An efficient resource sharing strategy between real-time voice and video calls in multimedia wireless networks was proposed and studied in this paper. Both analytical and simulation results for various channel borrowing policies were obtained and compared. We found that the proposed strategy can increase the radio channel utilization by carrying more voice traffic. As a result, the voice call blocking probability can be significantly reduced while the QoS requirement on video calls is not violated.

The borrowing concept proposed in this paper can also be used for giving priority to different classes of traffic. One poten- 
tial application is for prioritized handoff scheme using channel reservation for handoff calls [7]. The new call blocking probability can be cut down by allowing the reserved channels for handoff calls to be temporarily borrowed by new calls.

\section{ACKNOWLEDGMENT}

The authors would like to thank the comments and suggestions from the anonymous reviewers.

\section{REFERENCES}

[1] T. S. P. Yum and M. Chen, "Dynamic channel assignment in integrated services cable networks," IEEE Trans. Commun., vol. 42, no. 2/3/4, pp. 2023-2027, 1994.

[2] A. Iera, S. Marano, and A. Molinaro, "Transport and control issues in multimedia wireless networks," Wireless Networks, no. 2, pp. 249-261, 1996.

[3] G. M. Stamatelos and V. N. Koukoulidis, "Reservation-based bandwidth allocation in a radio ATM network," IEEE/ACM Trans. Networking, vol. 5, no. 3, pp. 420-428, June 1997.

[4] X. W. Wu et al., "Dynamic slot allocation multiple access protocol for wireless ATM networks," in Proc. IEEE Int. Conf. Commun.(ICC'97), Montreal, Quebec, June 1997, pp. 1560-1565.

[5] S. K. Biswas and D. Reininger, "Bandwidth Allocation for VBR Video in Wireless ATM Networks,", NEC USA C\&C Technical Report, May 1996.

[6] K. L. Yeung and T. S. P. Yum, "Cell group decoupling analysis of a dynamic channel assignment strategy in linear microcellular radio systems," IEEE Tran. Commun., vol. 43, no. 2/3/4, pp. 1289-1292, 1995.

[7] — , "Optimal prioritized handoff in linear microcellular radio systems," in Proc. IEEE GLOBECOM, Singapore, Nov. 1995, pp. 494-498.

[8] J. E. Wieselthier and A. Ephremides, "Fixed amd movable-boundary channel-access schemes for integrated voice/data wireless networks," IEEE Trans. Commun., vol. 43, pp. 64-74, Jan. 1995.

[9] B. Kraimeche and M. Schwartz, "Analysis of traffic control strategies in integrated service networks," IEEE Trans. Commun., vol. COM-33, pp. 1085-1093, Oct. 1985.

[10] M. Ostrowski, "Efficient transmission of integrated voice and data in wireless networks," in Proc. ICC'96, June 1996, pp. 721-727.

[11] H. Koraitim and S. Tohme, "Resource allocation and connection admission control in satellite networks," IEEE J. Select. Areas Commun., vol. 17, pp. 360-372, Feb. 1999.

[12] B. Kraimeche and M. Schwartz, "Bandwidth allocation strategies in wide-band integrated networks," IEEE J. Select. Area. Commun., vol. JSAC-4, pp. 869-878, Sept. 1986.

[13] - "A channel access structure for wideband," IEEE J. Select. Areas Commun., vol. JSAC-5, pp. 1327-1335, Oct. 1987.
[14] Y. H. Kim et al., "Analysis of bandwidth allocation strategies with access restriction in B-ISDN," IEEE Trans. Commun., vol. 41, pp. 771-781, May 1993.

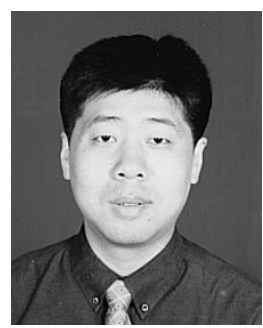

Xiaowen Wu (S'95-M'98) received the B.S. degree in electrical engineering, and the M.S and Ph.D. degrees in telecommunications and electronic engineering, all from the University of Electronic Science and Technology of China (UESTC), in 1989, 1995, and 1997, respectively.

From 1989 to 1992, he worked for the Southwest Communications Research Institute in China, where he engaged in research on N-ISDN, X.25 communications network, and satellite communications. From 1997 to 1998 , he was with Department of Electronic Engineering, City University of Hong Kong as a full-time research staff. His research interests are in third generation mobile system, wireless ATM networks, as well as ATM switching. Since 1998, he has been with ZTE Corporation of China, where he is the Chief Engineer of $3 \mathrm{G}$ product and leads a R\&D group to implement WCDMA system.

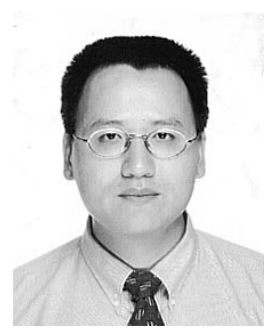

Kwan L. Yeung (M'95-SM'99) received the B.Eng. and $\mathrm{Ph} . \mathrm{D}$. degrees in information engineering from The Chinese University of Hong Kong in 1992 and 1995 , respectively.

In July 2000, he joined the of Department Electrical and Electronic Engineering, The University of Hong Kong. Before that, he has spent five years at the Department of Electronic Engineering at City University of Hong Kong as an Assistant Professor. His main research interests include personal and mobile communication systems, high-speed networking, broadband packet switches, and lightwave networks.

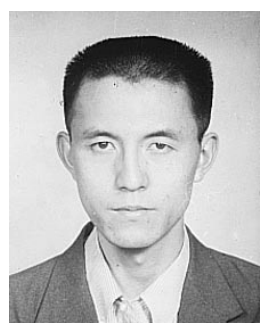

Jianhao Hu received the B.Eng and Ph.D. degrees in telecommunication from University of Electronic Science and Technology of China in 1993 and 1999, respectively.

He worked as Lecturer in the Institute of Information and System of UESTC from 1993 to 1998 . He is currently a a full-time research staff in City University of Hong Kong. His research interests include personal and mobile communication system, satellite communication, and high-speed networks. 